\title{
A Metabonomic Study on Samples of Cutaneous Leishmaniasis and its Correlation with the Selenium Level in the Blood Serum
}

\author{
Tafazzoli Mohsen ${ }^{1 *}$, Fathi Fariba1, Darvizeh Fatemeh ${ }^{1}$, Zahra Zamani $^{2}$ and Pourfallah Fatemeh ${ }^{2}$ \\ ${ }^{1}$ Department of Chemistry, Sharif University of Technology, Tehran, Iran \\ ${ }^{2}$ Pasteur Institute of Iran, Tehran, Iran
}

\begin{abstract}
This paper presents the result of extensive experiments on the blood serum samples of 20 leishmaniasis patients and 44 healthy individuals using nuclear magnetic resonance (NMR) spectroscopy and the Chenomx software. The concentrations of selenium in all 64 serum samples were also measured using an atomic absorption device. The healthy and patient groups were completely differentiated using the partial least square method (PLS). In addition, important variables that could highly influence the group of patients were detected by the PLS loading plot. This paper also presents the results corresponding to linear and non-linear modeling of selenium concentration in serum. Stepwise multiple linear regression (MLR) was used to select the five most important descriptors. In the multiple linear regression modeling approach the results obtained for $R^{2}$ training, test and validation sets were $0.98,0.97$ and 0.94 respectively. Employing the same descriptors in the MLR modeling approach, a non-linear artificial neural network (ANN) with a 5-31 structure was constructed; the results obtained from this model showed no significant improvement compared with those of MLR. There was good agreement between the experimental values of selenium concentration and the values from the two models.
\end{abstract}

Keywords: Cutaneous leishmaniasis; Nuclear magnetic resonance; Partial least square; Multiple linear regression; Artificial neural networks

Abbreviations: LV: Latent Variable; OSC: Orthogonal Signal Correction; PCA: Principal Components Analysis; PLS: Partial Least Squares; MLR: Multiple Linear Regression; ANN: Artificial Neural Networks; NMR: Nuclear Magnetic Resonance; CL: Cutaneous Leishmaniasis.

\section{Introduction}

${ }^{1} \mathrm{H}$ NMR spectroscopy of biofluids (urine, serum/plasma) and tissue generates comprehensive biochemical profiles of low-molecularweight endogenous metabolites [1]. NMR spectroscopy has emerged as a key tool for understanding metabolic processes in living systems [2]. Metabonomics is formally defined as "the quantitative measurement of the multi-parametric metabolic response of living systems to pathophysiological stimuli or genetic modification" [3,4]. This approach is complementary to proteomics and genomics and is applicable to a wide range of problems in diverse biomedical research areas. The applied ranges of spectroscopic techniques for metabonomics are often used in a so-called "hyphenated" mode (e.g., LC-NMR-MS); however, NMR-based metabonomics has proven to be particularly appropriate for the rapid analysis of complex biological samples [5-8]. Detailed inspection of NMR spectra and integration of individual peaks can give valuable information on dominant biochemical changes. Moreover, pattern-recognition methods can be used to map the NMR spectra into a lower dimensional space (than that implied by the number of points in the digital representation of the NMR spectrum), making it easier to determine any similarities of biochemical profiles among samples and to determine the biochemical basis for these similarities [5].

Leishmaniasis is a parasitic disease that is found in 88 countries throughout Africa, Asia, Europe and North and South America [9]. There are an estimated 12 million cases worldwide, with 1.5 to2 million new cases each year [9].The disease is caused by leishmania parasites, which are spread by the bite of infected sand flies. There are several different forms of leishmaniasis in the human; the most common is $\mathrm{CL}$, which causes skin sores.

Endemic CL is still considered an important health problem in many parts of the world, especially the Mediterranean region and almost all countries of the Middle East, including Iran [10]. For most infectious diseases, increased formation of reactive oxygen species is secondary to the primary disease process. Some microorganisms are highly susceptible to exogenous reactive oxygen species such as hydrogen peroxide [11].Glutathione peroxidase (GSH-Px) removes $\mathrm{H}_{2} \mathrm{O}_{2}$ and selenium is required for the activity of GSH-Px. Therefore, to assess the status of GSH-Px and selenium in CL, the concentration of selenium in the serum of 20 patients with $\mathrm{CL}$ and 44 healthy controls was determined (Table 1). Selenium concentrations were found to be lower in the patient group than in the controls $(\mathrm{p}<0.0001)$ [11]. Selenium has an important role in the pathophysiological processes of $\mathrm{CL}$ and decreased selenium levels may be a host defense strategy against $\mathrm{CL}$ infection. The NMR spectral profiles resulting from this

\begin{tabular}{|l|l|}
\hline Patient group & healthy group \\
\hline${ }_{ \pm} S D: 59.2 \pm 16.2(\mu \mathrm{g} / \mathrm{L})$ Mean & $\pm S D 81.8 \pm 13.7(\mu \mathrm{g} / \mathrm{L})$ Mean \\
\hline
\end{tabular}

Table 1: Concentrations of selenium for patients and healthy controls.

*Corresponding author: Mohsen Tafazzoli , Department of Chemistry, Sharif University of Technology, Tehran, Iran, Tel: +98 21 66165305; fax: +98 21 66012983; E-mail: Tafazzoli@sharif.edu

Received August 24, 2010; Accepted October 25, 2010; Published October 27 2010

Citation: Mohsen T, Fariba F, Fatemeh D, Zamani Z, Fatemeh P (2010) A Metabonomic Study on Samples of Cutaneous Leishmaniasis and its Correlation with the Selenium Level in the Blood Serum. J Mol Biomark Diagn 1:101. doi:10.4172/2155-9929.1000101

Copyright: @ 2010 Mohsen T, et al. This is an open-access article distributed under the terms of the Creative Commons Attribution License, which permits unrestricted use, distribution, and reproduction in any medium, provided the original author and source are credited. 
analysis, however, are complex and more easily interpreted with automated data reduction and chemometric analysis [4] such as PLS, ANN and MLR. PLS is a supervised classification method that groups the dataset into two clusters: healthy and patient samples. MLR is a common method for variable selection; it uses forward selection and backward deletion. ANN is a non-linear method for developing a robust model between the response and selected variants.

\section{Materials and Methods}

\section{Sample collection}

Blood was drawn from 20 leishmaniasis-positive patients attending the Pasteur Institute of Iran in Tehran. Samples were allowed to clot in non-anti-coagulant Vacutainer test tubes for two hours at room temperature (RT) and the serum was separated after centrifugation for 10 minutes at $2500 \mathrm{rpm}$ and RT. Aliquots of the serum were stored at $-80^{\circ} \mathrm{C}$ until assayed.

\section{${ }^{1}$ HNMR spectroscopy}

Prior to NMR analysis, serum samples $(600 \mu \mathrm{L})$ were diluted with $60 \mu \mathrm{L}$ of $52 \% \mathrm{D}_{2} \mathrm{O}$ (deuterium oxide, $99.9 \% \mathrm{D}$, Aldrich Chemicals Company, Wisconsin, USA) and placed in $5 \mathrm{~mm}$ high-quality NMR tubes (Sigma Aldrich, RSA). Conventional ${ }^{1} \mathrm{H}$ NMR spectra of each serum sample were measured at $500 \mathrm{MHz}$ on a Bruker DRX NMR instrument laboratory with Carr-Purcell-Meiboom-Gill (CPMG) method [10]. ${ }^{1} \mathrm{H}$ NMR spectra were recorded at $300 \mathrm{~K}$. For each spectrum, 200 scans were collected into $32 \mathrm{~K}$ computer data points with $8389 \mathrm{~Hz}$ width for spectra and relaxation delay time $1.5 \mathrm{~s}$. Metabolites were assigned on the basis of their chemical shifts and signal multiplicity.

\section{Atomic absorption spectrophotometer}

The level of selenium in each serum sample was determined by a Spectra AA 220 Plus Zeeman atomic absorption spectrophotometer with a graphite furnace GTA-110 (Varian, Australia), with deuterium background correction. Varian hollow cathode lamps were employed at $196 \mathrm{~nm}$ wavelength and $1.0 \mathrm{~nm}$ band pass. Pyrolytically coated graphite tubes with pyrolytic graphite platforms (Varian) were used. Selenium concentration was determined by an internal standard addition method as previously described [12]. The serum was diluted (1:4) with $0.05 \%$ Triton-X100 in $0.125 \%$ nitric acid. All determinations were run in duplicate and individual values were averaged. By means of an auto sampler, $10 \mu \mathrm{L}$ of the solution was dispensed on the atomizer platform, together with $10 \mu \mathrm{L}$ of $1 \mathrm{mg} / \mathrm{mL}$ palladiumchloride and $2 \mathrm{~mL} 2 \%(\mathrm{w} / \mathrm{v})$ ascorbic-acid solution.

\section{Data reduction of NMR data}

All plasma ${ }^{1} \mathrm{HNMR}$ spectra were manually phased and baseline corrected using the Chenomx NMR suite (version 6.0). The 0.010.0 parts per million $(\mathrm{ppm})$ spectral regions were reduced to 250 integral segments of an equal width of $0.04 \mathrm{ppm}$. The integrals were measured for each of the 250 regions (Figure 1) and the spectral region containing the water resonance $(4.6-5.0 \mathrm{ppm})$ was removed from each subdivided spectrum, thereby eliminating spectral variations due to differences in water suppression from one spectrum to the next $[5,13,14]$. This optimal width of segmented regions is based on previous studies, which found that regions of $0.04 \mathrm{ppm}$ accommodated any small pH-related shifts in signals and variation in shimming quality [5]. The integral values of each spectral region were normalized to a constant sum of all integrals in a spectrum to reduce any significant concentration differences between samples $[13,14]$.

\section{Statistical analysis}

Orthogonal signal correction (OSC): OSC, a preprocessing step to NMR spectroscopy analysis, removes orthogonal variations in the class of interest. Because of its powerful attributes, it has received increased emphasis in metabonomics studies. OSC can essentially filter any confounding factors that obscure interesting biological variations $[15,16]$. In the OSC procedure, the identity of the sample classes is included in the calculation and designated by a vector, Y. The first step in the OSC procedure calculates the first principal component or score vector, $t$, an optimal linear description of the spectral data; it describes maximum separation based on class.

The longest vector orthogonal to $\mathrm{Y}$ is calculated; and this vector, $t^{*}$, describes the greatest source of variation that is not related to class yet still provides a good summary of the spectral data [17]. This is done as:

$$
\left(1-Y\left(Y^{T} Y\right)^{-1} Y^{T}\right) t=t^{*}
$$

Next, the loading vector, $\mathrm{p}^{*}$, relating to this corrected vector is calculated and the product of the orthogonal score and loading vectors is subtracted from the spectral data. Examination of the OSC scores, $t^{*}$ and loadings $p^{*}$ can be useful in determining the source of the removed variation. The residual matrix represents the filtered spectral data and is used for the calculation of PLS.

PLS: The PLS model was calculated and graphed using MATLAB. PLS uses both an X-matrix and a Y-matrix; $\mathrm{Y}$ in this case is a vector describing the concentration of the denaturant. The $\mathrm{X}$ matrix is decomposed into a set of orthogonal components, but instead of describing the maximum variance in $X$ (as in PCA), these components describe the maximum covariance between $X$ and $Y(P L S)$. The scores, $\mathrm{T}$, relate $\mathrm{X}$ and $\mathrm{Y}$ to each other. For each component in PLS, $\mathrm{Y}$ is described as a linear combination of all the $X$ variables. The weight for each component, $\mathrm{w}$, describes how important a certain variable is for describing the response.

Multiple linear regression: The general purpose of multiple linear regression is to quantify the relationship between several independent or predictor variables and a dependent variable. In this study, a set of coefficients defined the single linear combination of independent variables (molecular descriptors) that best described selenium levels. The selenium concentration for each serum sample was calculated as a composite of each molecular descriptor weighted by the respective coefficients. Initially stepwise MLR was applied to select the best descriptors among 250 variables. The selected variables were used to make a multi linear model. The resulting multi linear model can be represented as:

$$
\mathrm{y}=\beta_{0}+\beta_{1} \mathrm{X}_{1}+\beta_{2} \mathrm{X}_{2}+\beta_{3} \mathrm{X}_{3}+\ldots+\beta_{\mathrm{k}} \mathrm{X}_{\mathrm{k}}+\varepsilon
$$

where $k$ is the number of independent variables and $y$ is the dependent variable. Regression coefficients represent the independent contributions of each calculated molecular descriptor. The matrix notation of algebraic MLR model is defined as below:

$$
\begin{aligned}
& y=X b^{\wedge}+e \\
& b^{\wedge}=\left(X^{T} X\right)^{-1} X^{T} y
\end{aligned}
$$

where $b^{\wedge}$ is the estimator for the regression coefficients. The MLR model was built using a training set and validated by an external prediction set. Multiple linear regression (MLR) techniques based on least-squares procedures are often used for estimating the coefficients involved in the model equation [18]. 

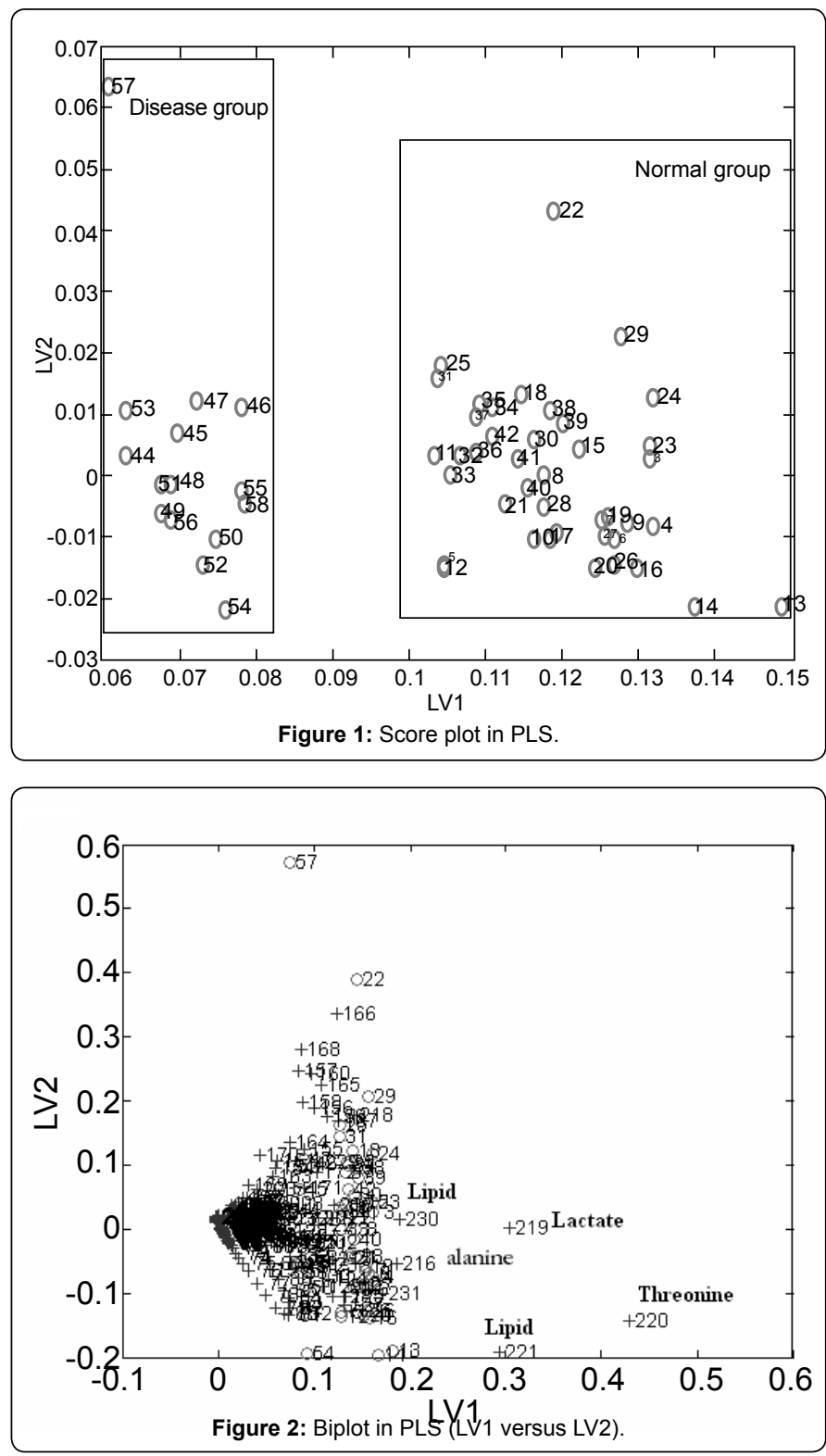

Artificial neural networks model: The theory behind artificial neural networks has been described in detail elsewhere [19]. The current study designed a three-layer back-propagation network with a sigmoidal transfer function, written in MATLAB in our laboratory. The three descriptors appearing in our previous QSMR model were used as input parameters for generating the network. The signals from the output layer represented the selenium concentration. Such an ANN may be designed as a 5-3-1 net to indicate the number of nodes in input, hidden and output layers, respectively. Generally, the neural network methodology has several empirically determined parameters. These include: when to stop training (i.e. the number of iterations or the convergence criterion), the number of hidden nodes and the learning rate and momentum terms. The values of constructed ANN parameters were optimized using the procedure that was reported in our previous works [20-22]. The initial weights were chosen randomly and the program was written so that the randomized weights depended on the number of input, hidden and output nodes. To evaluate the performance of the ANN, standard error of calibration (SEC) and standard error of prediction (SEP) were used [23]. The number of neurons in the hidden layer, with a minimum value of SEC, was selected as the optimum number. Learning rate and momentum were similarly optimized. The validation set was used to examine the validity of the ANN model.

\section{Result and Discussion}

PCA was primarily used for distinguishing outliers [24]. Consequently, the number of samples decreased to 58 (16 patients and 42 healthy individuals). The PLS was employed as a supervised method to classify the patient and healthy groups based on the selenium concentration. Figure 1 shows the results of classification of the two-dimensional score plot with two latent variables. In addition, the PLS plots (Figure 2 and Figure 3 ) depict the importance of variables selected by the stepwise MLR method. As shown in Table 3, the loading plot confirmed that threonine, lipids, lactate and alanine were the most important variables. The importance of metabolites determined in this study, supported by the literature [25-30], confirmed our results. For the next step, modeling selenium levels based on the five best descriptors was the main goal. Table 2 shows the MLR-calculated values of selenium concentration for all serum samples. Table 4 presents the regression results for the selected MLR model. The $\mathrm{R}^{2}$ for training, test and validation sets were found to be $0.98,0.97$ and 0.94 respectively. Figure 4 shows the correlation between the calculated MLR and the experimental values for selenium levels included in the test and validation sets. The correlation of $\mathrm{R}^{2}=0.95$ (test and validation sets) indicated a reasonable agreement between these values.

Developing an ANN and comparing it with the MLR models

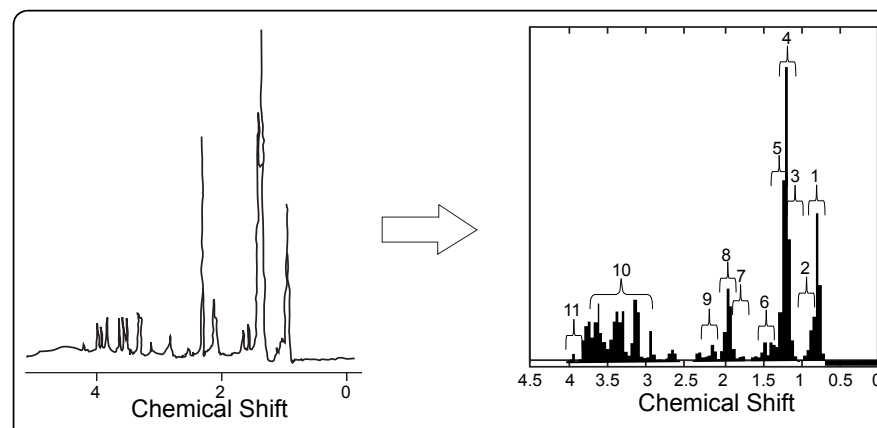

Figure 3: Data reduction of the region of a ${ }^{1} \mathrm{H}$ NMR spectrum of a bio-fluid sample into spectral regions of equal width in ppm.[1] lipids, [2] valine/ isoleucine, [3] lipids, [4] threonine, [5] lactate, [6] alanine,[7] arginine/lysine, [8] proline,[9] glutamine,[10] glucose/amino acids, [11] lactate.

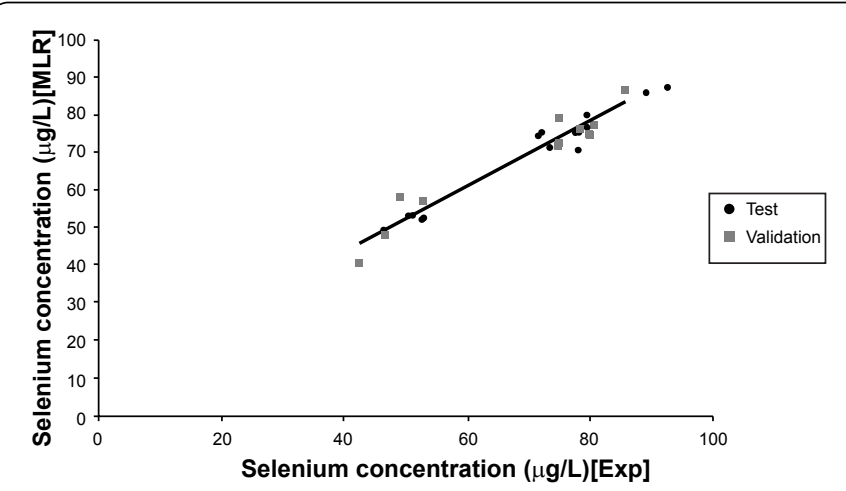

Figure 4: Plot of the MLR calculated selenium against the experimental values of selenium concentration for the test and validation sets. 


\begin{tabular}{|c|c|c|c|c|c|c|c|c|}
\hline \multicolumn{9}{|c|}{ Descriptors } \\
\hline No. & $\mathrm{Y}$ & Threonine & Lipid & Lactate & Alanine & Lipid & $A N N$ & $M L R$ \\
\hline \multicolumn{9}{|c|}{ Training set } \\
\hline 1 & 75.80 & 0.03 & 0.04 & 0.01 & 0.01 & 0.01 & 74.49 & 73.80 \\
\hline 3 & 88.70 & 0.04 & 0.06 & 0.01 & 0.01 & 0.01 & 87.55 & 87.06 \\
\hline 5 & 70.40 & 0.03 & 0.04 & 0.01 & 0.01 & 0.01 & 70.30 & 72.34 \\
\hline 7 & 84.50 & 0.03 & 0.06 & 0.01 & 0.01 & 0.01 & 83.44 & 84.41 \\
\hline 9 & 86.40 & 0.04 & 0.06 & 0.01 & 0.01 & 0.01 & 85.82 & 89.04 \\
\hline 11 & 69.90 & 0.03 & 0.04 & 0.01 & 0.01 & 0.01 & 70.80 & 70.86 \\
\hline 13 & 100.30 & 0.05 & 0.06 & 0.02 & 0.01 & 0.02 & 99.5 & 103.13 \\
\hline 15 & 82.50 & 0.04 & 0.06 & 0.01 & 0.01 & 0.01 & 83.59 & 84.18 \\
\hline 17 & 80.00 & 0.03 & 0.05 & 0.01 & 0.01 & 0.01 & 81.27 & 79.49 \\
\hline 19 & 85.00 & 0.04 & 0.05 & 0.02 & 0.01 & 0.01 & 85.55 & 85.49 \\
\hline 21 & 75.80 & 0.03 & 0.05 & 0.01 & 0.01 & 0.01 & 76.38 & 75.01 \\
\hline 23 & 88.70 & 0.03 & 0.05 & 0.01 & 0.01 & 0.02 & 89.33 & 83.91 \\
\hline 25 & 70.40 & 0.04 & 0.03 & 0.01 & 0.01 & 0.01 & 69.8 & 73.07 \\
\hline 27 & 84.50 & 0.04 & 0.05 & 0.01 & 0.01 & 0.01 & 84.38 & 83.50 \\
\hline 29 & 86.40 & 0.04 & 0.05 & 0.01 & 0.02 & 0.01 & 85.22 & 89.03 \\
\hline 31 & 70.10 & 0.03 & 0.03 & 0.01 & 0.01 & 0.01 & 72.20 & 70.42 \\
\hline 33 & 71.00 & 0.03 & 0.04 & 0.01 & 0.01 & 0.01 & 68.58 & 69.43 \\
\hline 35 & 74.00 & 0.03 & 0.06 & 0.01 & 0.01 & 0.00 & 74.18 & 72.30 \\
\hline 37 & 73.40 & 0.03 & 0.04 & 0.01 & 0.01 & 0.01 & 75.76 & 70.57 \\
\hline 39 & 81.00 & 0.04 & 0.05 & 0.01 & 0.01 & 0.01 & 81.40 & 82.69 \\
\hline 41 & 77.00 & 0.04 & 0.05 & 0.02 & 0.01 & 0.00 & 76.15 & 78.01 \\
\hline 43 & 45.70 & 0.02 & 0.03 & 0.01 & 0.01 & 0.00 & 44.38 & 47.39 \\
\hline 45 & 47.20 & 0.03 & 0.03 & 0.00 & 0.00 & 0.00 & 46.90 & 47.60 \\
\hline 47 & 49.10 & 0.03 & 0.02 & 0.01 & 0.01 & 0.00 & 49.30 & 47.75 \\
\hline 49 & 45.50 & 0.02 & 0.02 & 0.01 & 0.01 & 0.01 & 44.01 & 50.83 \\
\hline 51 & 45.50 & 0.02 & 0.03 & 0.01 & 0.00 & 0.01 & 46.87 & 45.00 \\
\hline 53 & 42.60 & 0.00 & 0.05 & 0.01 & 0.01 & 0.01 & 43.67 & 44.85 \\
\hline 55 & 52.80 & 0.02 & 0.04 & 0.01 & 0.00 & 0.00 & 51.90 & 54.20 \\
\hline 57 & 41.50 & 0.02 & 0.02 & 0.00 & 0.01 & 0.00 & 43.29 & 39.32 \\
\hline 2 & 80.60 & 0.03 & 0.05 & 0.03 & 0.00 & 0.00 & 81.64 & 83.21 \\
\hline 6 & 85.60 & 0.04 & 0.06 & 0.02 & 0.01 & 0.01 & 86.49 & 87.44 \\
\hline 12 & 70.20 & 0.03 & 0.04 & 0.01 & 0.01 & 0.01 & 69.52 & 71.59 \\
\hline 14 & 87.50 & 0.03 & 0.07 & 0.02 & 0.01 & 0.01 & 86.19 & 85.73 \\
\hline 20 & 84.00 & 0.03 & 0.06 & 0.02 & 0.01 & 0.01 & 85.11 & 83.87 \\
\hline 24 & 89.10 & 0.04 & 0.06 & 0.01 & 0.01 & 0.01 & 89.98 & 84.77 \\
\hline \multicolumn{9}{|c|}{ Test set } \\
\hline 28 & 79.30 & 0.04 & 0.06 & 0.01 & 0.01 & 0.01 & 78.24 & 80.03 \\
\hline 32 & 72.00 & 0.03 & 0.05 & 0.01 & 0.01 & 0.01 & 74.80 & 74.88 \\
\hline 36 & 73.50 & 0.03 & 0.05 & 0.01 & 0.01 & 0.00 & 72.94 & 71.12 \\
\hline 40 & 78.00 & 0.04 & 0.04 & 0.02 & 0.01 & 0.01 & 79.20 & 70.65 \\
\hline 46 & 52.80 & 0.03 & 0.03 & 0.00 & 0.01 & 0.00 & 49.13 & 52.55 \\
\hline 50 & 50.50 & 0.02 & 0.03 & 0.01 & 0.00 & 0.01 & 53.70 & 53.07 \\
\hline 54 & 51.00 & 0.02 & 0.04 & 0.01 & 0.00 & 0.01 & 51.35 & 53.28 \\
\hline 58 & 46.50 & 0.02 & 0.03 & 0.01 & 0.00 & 0.01 & 47.09 & 49.00 \\
\hline 4 & 89.10 & 0.04 & 0.05 & 0.02 & 0.01 & 0.01 & 89.80 & 86.04 \\
\hline 8 & 79.30 & 0.03 & 0.05 & 0.02 & 0.01 & 0.01 & 78.04 & 76.68 \\
\hline 10 & 78.10 & 0.03 & 0.05 & 0.01 & 0.01 & 0.01 & 78.60 & 75.30 \\
\hline 14 & 92.50 & 0.04 & 0.06 & 0.02 & 0.01 & 0.01 & 90.72 & 87.33 \\
\hline 18 & 77.50 & 0.04 & 0.04 & 0.01 & 0.01 & 0.01 & 77.37 & 75.48 \\
\hline \multicolumn{9}{|c|}{ Validation set } \\
\hline 22 & 80.60 & 0.03 & 0.05 & 0.01 & 0.02 & 0.00 & 84.3 & 77.29 \\
\hline 26 & 85.60 & 0.04 & 0.05 & 0.02 & 0.01 & 0.02 & 84.76 & 86.13 \\
\hline 30 & 78.40 & 0.03 & 0.05 & 0.01 & 0.01 & 0.01 & 71.62 & 76.34 \\
\hline 32 & 75.00 & 0.03 & 0.05 & 0.01 & 0.01 & 0.00 & 73.26 & 72.15 \\
\hline 38 & 80.00 & 0.04 & 0.04 & 0.02 & 0.01 & 0.01 & 79.10 & 74.84 \\
\hline 42 & 75.00 & 0.04 & 0.05 & 0.01 & 0.01 & 0.01 & 75.85 & 79.10 \\
\hline 44 & 42.60 & 0.01 & 0.03 & 0.01 & 0.01 & 0.00 & 41.30 & 40.32 \\
\hline 48 & 46.50 & 0.01 & 0.04 & 0.01 & 0.00 & 0.01 & 42.49 & 48.58 \\
\hline 52 & 49.10 & 0.03 & 0.03 & 0.01 & 0.00 & 0.00 & 49.42 & 58.14 \\
\hline 56 & 52.80 & 0.03 & 0.03 & 0.01 & 0.01 & 0.01 & 47.51 & 56.83 \\
\hline
\end{tabular}

Table 2: The results of MLR and ANN modeling and the values of descriptors.

provided the opportunity to investigate the nonlinear characteristics of the dependence of selenium concentration on metabolites. In order to have a meaningful comparison, the variables for the linear and nonlinear treatments should be the same. Therefore, the five descriptors appearing in the MLR model were considered as the inputs for generating the networks. After optimizing the parameters for constructing the artificial neural network, the structure of 5-3-1 was obtained. A test set of twenty serum samples was used to optimize the learning iteration size and avoid overtraining. To evaluate the network, the concentrations of selenium included in the validation set were predicted (Table 2). Figure 5 shows the plot of the ANNpredicted versus the experimental values for selenium concentration, illustrating that the validation sets showed better values than those obtained by the MLR method.

Figure 6 shows the predicted values for the residuals of ANN for different selenium concentrations as well as the experimental values. 


\begin{tabular}{|l|l|l|l|}
\hline Descriptor & assignment & Coefficient & Mean effect $^{\text {a }}$ \\
\hline Threonine & $\mathrm{YCH}_{3}$ & 811.61 & 25.97 \\
\hline Lipid & $\mathrm{CH}_{3} \mathrm{CH}_{2} \mathrm{CH}_{2}$ & 455.19 & 20.58 \\
\hline Lactate & $\mathrm{CH}_{3}$ & 610.94 & 7.33 \\
\hline Alanine & $\mathrm{CH}_{3}$ & 837.68 & 6.62 \\
\hline Lipid & $\mathrm{CH}_{3} \mathrm{CH}_{2}\left(\mathrm{CH}_{2}\right)$ & 585.91 & 4.45 \\
\hline
\end{tabular}

aMean effect of a descriptor is the product of its mean and regression coefficient in the MLR model

Table 3: Specifications of the selected MLR model.

\begin{tabular}{|c|c|c|c|c|c|c|c|c|c|c|}
\hline & \multicolumn{3}{|c|}{ Training set } & \multicolumn{3}{|c|}{ Test Set } & \multicolumn{3}{|c|}{ Validation set } & Total \\
\hline & RMSE & $\mathrm{R}^{2}$ & F-test & RMSE & $\mathrm{R}^{2}$ & F-test & RMSE & $\mathrm{R}^{2}$ & F-test & $\mathrm{Q}^{2}(\mathrm{LOO})$ \\
\hline MLR & 2.19 & 0.98 & 8525.60 & 2.34 & 0.97 & 372.60 & 4.06 & 0.94 & 256.77 & 0.95 \\
\hline ANN & 1.17 & 0.99 & 6447.50 & 1.90 & 0.98 & 760.86 & 3.15 & 0.97 & 115.03 & 0.97 \\
\hline
\end{tabular}

Table 4: Comparison of the statistics for the MLR ANN models.

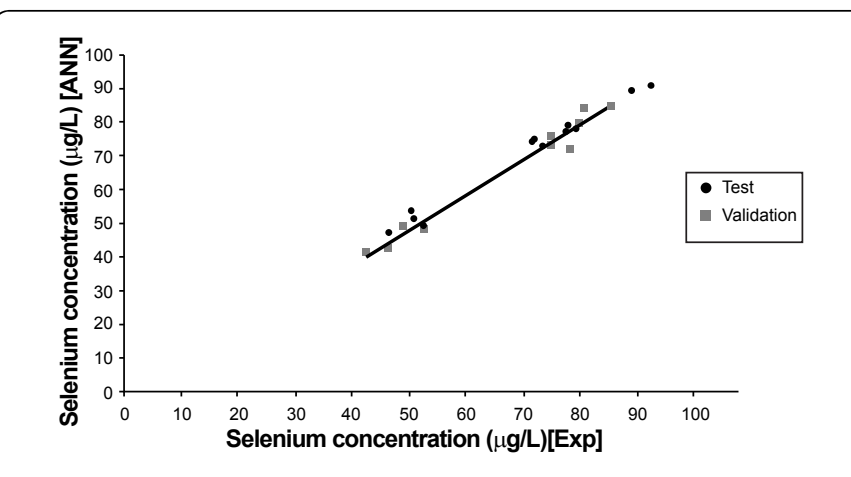

Figure 5: Plot of the ANN calculated selenium against the experimental values of selenium concentration for the test and validation set.

The propagation of the residuals on both the positive and negative sides of the $\mathrm{x}$ axis indicates that there was no systematic error in the development of the neural network.

Table 4 lists in more detail data about the predictive power of the MLR and ANN models. The data suggests that linear and non-linear models were in good agreement with the experimental results. The ANN-predicted values also showed much lower standard errors when compared with those of the MLR model. The ANN model also revealed a higher value for the F-statistic. In order to prove the validity and stability of the models, cross-validation tests using the leave-one-out method were performed; Table 4 summarizes the results.

\section{Conclusion}

Results using both ANN and MLR clearly show that the two regions of selenium concentration below and above $60 \mu \mathrm{g} / \mathrm{L}$ correspond to patient and healthy groups respectively. In finding the five most important descriptors, this study has demonstrated a significant relationship between selenium concentration and descriptors such as threonine, lipids, lactateand alanine. Moreover, PLS clearly classifies the data into two groups: patients and healthy. These five descriptors have a considerable role in modeling the selenium concentration of serum and dividing samples into two distinct groups.

\section{References}

1. Solanky KS, Bailey NJC, Beckwith-Hall BM, Davies A, Bingham S, et al. (2003) Application of biofluid $1 \mathrm{H}$ nuclear magnetic resonance-based metabonomic techniques for the analysis of the biochemical effects of dietary isoflavones on human plasma eseprofile. Anal Biochem 323:197-204.

2. Brown FF, Campbell ID, Kuchel PW, Rabenstein DL (1977) Human erythrocyte metabolism studies by $1 \mathrm{H}$ spin echo NMR. FEBS Lett 82: 12-16.

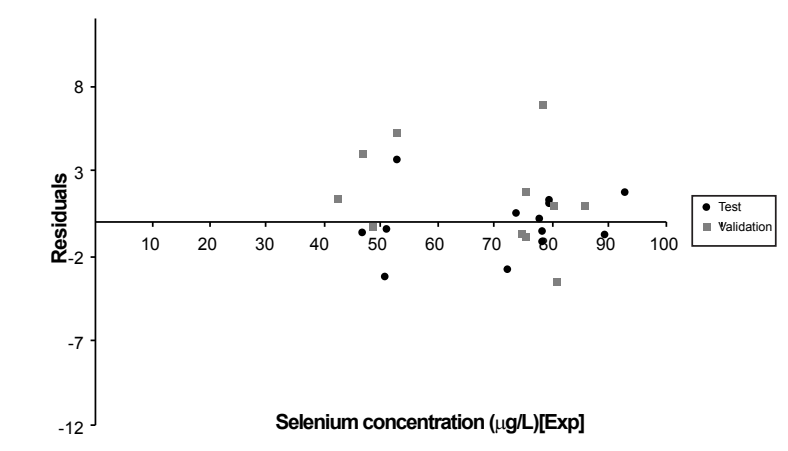

Figure 6: Plot of the residuals vs. the experimental values of selenium concentration.

3. Nicholson JK, Connelly J, Lindon JC, Holmes E (2002) Metabonomics: a platform for studying drug toxicity and gene function. Nat Rev Drug Discov 1:153-161.

4. Nicholson JK, Lindon JC, Holmes E (1999) 'Metabonomics': understanding the metabolic responses of living systems to pathophysiological stimuli via multivariate statistical analysis of biological NMR spectroscopic data. Xenobiotica 29: 1181-1189.

5. Coen M, Kuchel Ph (2004) Metabonomics based on NMR Spectroscopy. CiA 71: 13-17.

6. Odunsi K, Wollman RM, Ambrosone CB, Hutson A, McCann SE, et al. (2005) Detection of epithelial ovarian cancer using $1 \mathrm{H}-\mathrm{NMR}$-based metabonomics. Int J Cancer 113:782-788.

7. Pan Z, Gu H, Talaty N, Chen H, Shanaiah N, et al. (2007) Principal component analysis of urine metabolites detectedby NMR and DESI-MS in patients with inborn errors of metabolism. Anal Bioanal Chem 387: 539-549.

8. Serkova NJ, Niemann CU (2006) Pattern recognition and biomarker validation using quantitative $1 \mathrm{H}-\mathrm{NMR}$-based metabolomics. Expert Rev Mol Diagn 6: 717-731.

9. Report of the consultative meeting on cutaneous leishmanaisis, Geneva, WHO Head quarters, 2007.

10. Farahmand M, Assmar M, Nahrevanian H, Farzanehnejad Z, Piazak N (2008) Cutaneous leishmaniasis in patients referred to the Pasteur Institute of Iran during 2003-2006. The Internet Journal of Parasitic Diseases 3: 2.

11. Ozcan A, Gurel MS, Seyrek A, Aktepe N, Gur S, et al. (1999) Decreasing Selenium Levels and Glutathione Peroxidase Activity in Patients With Cutaneous Leishmaniasis. Tr. J. of Medical Sciences 29: 291-295.

12. Korunová V, Skodová Z, Dĕdina J, Valenta Z, Parizek J, et al. (1993) Serum selenium in adult Czechoslovak (Central Bohemia) population. Biol Trace Elem Res 37: 91-99.

13. Holmes E, Nicholis AW, Lindon JC, S. Ramos, Spraul M, et al. (1998) Development of amodel for classification of toxin-induced lesions using 1 HNMR spectroscopy of urine combined with pattern recognition. NMR Biomed 11: 235-244 
Citation: Mohsen T, Fariba F, Fatemeh D, Zamani Z, Fatemeh P (2010) A Metabonomic Study on Samples of Cutaneous Leishmaniasis and its Correlation with the Selenium Level in the Blood Serum. J Mol Biomark Diagn 1:101. doi:10.4172/2155-9929.1000101

14. Waters NJ, Holmes E, Williams A, Waterfield CJ, Farrant RD, et al. (2001) NMR and pattern recognition studies on the time-related metabolic effects of alpha-naphthylisothiocyanate on liver, urine and plasma in the rat: an integrative metabonomic approach. Chem Res Toxicol 14: 1401-1412.

15. Gavaghan C, Wilson ID, Nicholson JK (2002) Physiological variation in metabolic phenotyping and functional genomic studies: use of orthogonal signal correction and PLS-DA. FEBS Lett 530: 191-196.

16. Beckwith-Hall BM, Brindle JT, Barton RH, Coen M, Holmes E, et al. (2002) Application of orthogonal signal correction to minimise the effects of physical and biological variation in high resolution $1 \mathrm{H}$ NMR spectra of biofluids. Analyst 127: 1283-1288.

17. Wold S, Antti H, Lindgren F, Öhman J (1998) Orthogonal signal correction of near-infrared spectra. Chemometr Intell Lab 44: 175-185.

18. Martens H,Naes T (1989) Multivariate Calibration. Wiley: Chichester.

19. Hopke PK, Song X (1997) Classification of single particles by neural networks based on the computer-controlled scanning electron microscopy data. Anal Chim Acta 348: 375-388.

20. Jalali-Heravi M, Parastar F (2000) Development of comprehensive descriptors for multiple linear regression and artificial neural network modeling of retention behaviors of a variety of compounds on different stationary phases. J Chromatogr. A 903: 145-154.

21. Jalali-Heravi M, Garkani-Nejad Z (2001) Prediction of electrophoretic mobilities of sulfonamides in capillary zone electrophoresis using artificial neural networks. J Chromatogr A 927: 211-218.
22. Jalali-Heravi M, Kyani A (2007) Application of genetic algorithm-kernel partial least square as a novel nonlinear feature selection method: Activity of carbonic anhydrase II inhibitors. Eur J Med Chem 42: 649-659.

23. Blank TB, Brown ST (1993) Data processing using neural networks. Anal Chem 277: 273-287.

24. Psihogios NG, Gazi IF, Elisaf MS, Seferiadis KI, Bairak ET (2008) Genderrelated and age-related urinalysis of healthy subjects by NMR-based metabonomics. NMR Biomed 21: 195-207.

25. Yardley V, Croft SL (1997) Activity of Liposomal Amphotericin B Experimental again Cutaneous Leishmaniasis. AAC 41: 752-756.

26. Tappe D, Muller A, Stich A (2010) Resolution of Cutaneous Old World and New World Leishmaniasis after Oral Miltefosine Treatment. Am J Trop Med Hyg 82: 1-3.

27. Bansal D, Bhatti H.S, R. Sehgal (2005) Role of cholesterol in parasitic infections. Lipids Health Dis 4: 10.

28. Soong L(2008) Modulation of Dendritic Cell Function by Leishmania Parasites. J Immunol 180: 4355-4360.

29. Caceres A, Villaseca P, Claude J, Banuls AL, Inga R, et al. (2004) Epidemiology of Andean cutaneous leishmaniasis: incrimination of Lutzomyia ayacuchensis (Diptera: psychodidae) as a vector of Leishmania in geographically isolated, upland valleys of Peru. Am J Trop Med Hyg 70: 607-612.

30. Hashiguchi $Y$ (1996) Leishmaniasis: Its Changing Pattern and Importance as an Imported Disease. Intern Med 35: 434-435. 